\title{
Hypoglycaemia and severe plasmodium falciparum malaria among pregnant sudanese women in an area characterized by unstable malaria transmission
}

\author{
Aziem A Ali ${ }^{1}$, Elhassan M Elhassan², Mamoun M Magzoub ${ }^{1}$, Mustafa I Elbashir ${ }^{3}$ and Ishag Adam ${ }^{3 *}$
}

\begin{abstract}
Background: Pregnant women are more susceptible to severe Plasmodium falciparum malaria, which can lead to poor maternal and fetal outcomes. Few data exist on the epidemiology of severe $P$. falciparum malaria in pregnant women.

A hospital-based study was carried out to assess the pattern of severe $P$. falciparum malaria among pregnant women at the Kassala and Medani maternity hospitals, which are located in areas of unstable malaria transmission, in eastern and central Sudan, respectively. Pre-tested questionnaires were used to gather socio-demographic, clinical and obstetrical data. Suitable tests were performed for clinical and biochemical investigations.

Results: Among 222 pregnant women diagnosed with malaria at the two hospitals, 40 (18.0\%) women at mean (SD) gestational age of 29.3 (6.7) weeks fulfilled one or more of the WHO criteria for severe $P$. falciparum malaria. These were hypoglycaemia (14; 35.5\%), severe anaemia (12; 30\%), hypotension (10; 25\%), jaundice $(9 ; 22.5 \%)$, cerebral malaria $(6 ; 15 \%)$, repeated convulsions $(4 ; 10 \%)$, hyperparasitaemia $(4 ; 10.0 \%)$ and more than one manifestation $(9 ; 22.5 \%)$. While the mean (SD) presenting temperature was significantly lower for women presenting with hypoglycaemia [38.2(0.6) versus $\left.38.8(0.7){ }^{\circ} \mathrm{C}, P=0.04\right]$, other clinical and biochemical characteristics were not significantly different among women with different manifestations of severe $P$. falciparum malaria.
\end{abstract}

Conclusion: Preventive measures for pregnant women such as insecticide-treated bednets and chemoprophylaxis may be beneficial in areas of unstable malaria transmission. Early detection and prompt treatment of severe malaria, especially in pregnant women with hypoglycaemia, are needed.

\section{Background}

Malaria in pregnancy is a major public health problem in tropical and subtropical regions of the world. In Africa, millions of women living in malaria-endemic areas become pregnant each year [1,2]. Malaria in pregnancy contributes to significant maternal and perinatal morbidity and mortality. Each year, more than 500,000 women die during pregnancy or childbirth [1]. Severe malaria is a medical emergency associated with high mortality, especially in cases with multiple organ dysfunction [3]. Cerebral malaria and severe malarial

\footnotetext{
* Correspondence: ishagadam@hotmail.com

${ }^{3}$ Faculty of Medicine, University of Khartoum, Sudan

Full list of author information is available at the end of the article
}

anaemia are two major syndromes causing malariarelated mortality [4]. Children and pregnant women are the most vulnerable groups to the severe form of $P$. falciparum malaria [3]. While much literature and many publications are available on severe malaria in children, few published data exist on severe malaria during pregnancy [5]. Pregnant women are more attractive to the main malaria vector and the disease, including its severe form, than their non-pregnant counterparts [6-8]. Understanding the interactions that underlie the disease and its control should be helpful to investigate the epidemiology of severe malaria. Thus, such study is vital and may be of great interest for providing health planners and caregivers with fundamental guidelines for the implementation of preventive

\section{C) Biomed Central}


measures. In Sudan, the largest country in Africa, high maternal and perinatal mortality have been observed in different regions, and both malaria and anaemia were the major causes of these high levels of mortality [9-11].

Thus, the present study was conducted at the Kassala and Medani maternity hospitals in Sudan, which are located in areas characterized by unstable malaria transmission [12], and where malaria is a substantial burden affecting pregnant women irrespective of their age or parity [13].

\section{Methods}

This study was conducted at the Kassala and Medani (Figure 1) maternity hospitals in Sudan during the period from July to November 2010 for investigating the epidemiology of severe $P$. falciparum malaria among pregnant women. Pregnant women with symptoms of $P$. falciparum malaria were included in this study after informed consent was obtained from the patient or guardian. Those women with one or more of the manifestations of severe $P$. falciparum malaria according to the World Health Organization (WHO) criteria, which include cerebral malaria (unarousable coma), convulsion (more than two per 24 hours), hypotension (systolic blood pressure $<90 \mathrm{mmHg}$ with cold extremities), severe anaemia (haemoglobin $<7 \mathrm{gm} / \mathrm{dl}$ ), jaundice (detected clinically or bilirubin $>1 \mathrm{mg} / \mathrm{dl}$ ), hypoglycaemia (blood

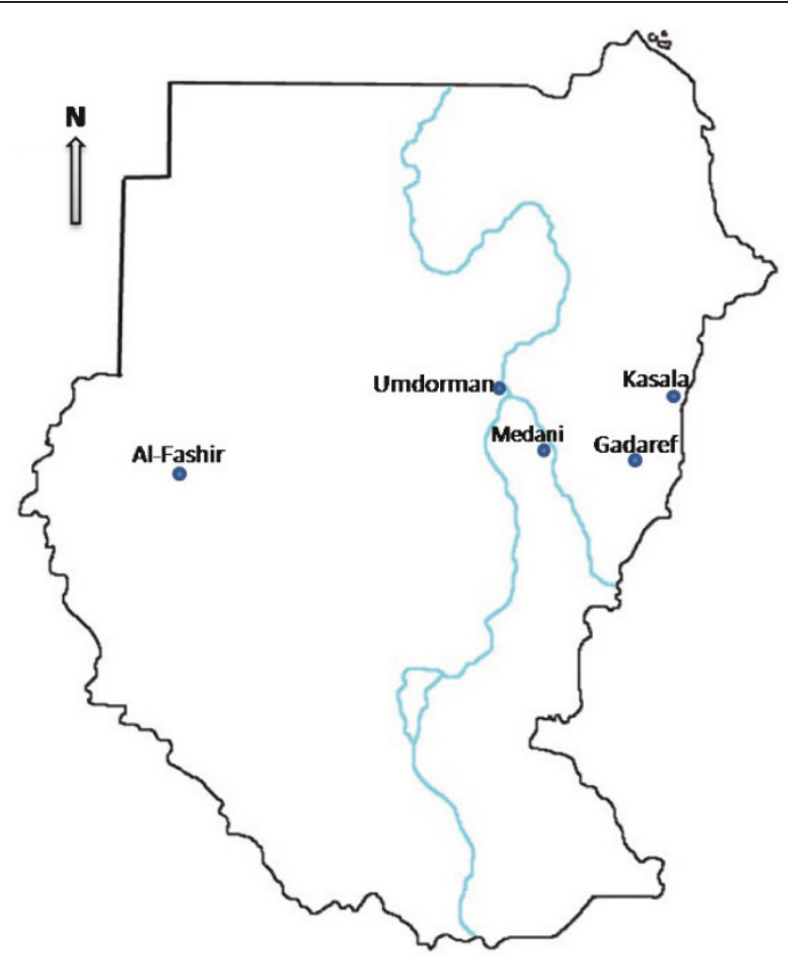

Figure 1 Map showing different regions of Sudan. glucose $<40 \mathrm{mg} / \mathrm{dl}$ ) and hyperparasitaemia (parasite count $>100,000$ ring forms $/ \mu \mathrm{l}$ ), were managed according to the WHO guidelines, and the rest were considered as uncomplicated cases [3]. Questionnaires were used to gather socio-demographic, medical and obstetrical data.

Blood films were prepared and stained with Giemsa, and 100 oil immersion fields were examined. The parasite density was evaluated by counting the number of asexual P. falciparum parasites for every 200 leukocytes, assuming a leukuocyte count of 8000 leukocytes/ $\mu \mathrm{l}$. All slides were double-checked in a blinded manner and only considered negative if no parasites were detected in 100 oil immersion fields. If gametocytes were seen, then the count was extended to 500 oil immersion fields. Haemoglobin concentrations were estimated using a HemoCue haemoglobinometer (HemoCue AB, Angelhom, Sweden). Blood glucose was measured at baseline before quinine infusion, two hours after quinine infusion and if there was clinical suspicion of hypoglycaemia using the bedside device Accu-Chek ${ }^{\mathrm{TM}}$ Multiclix (Roche diagnostics, Mannheim Germany). The Accu-Chek ${ }^{\mathrm{TM}}$ machine was calibrated weekly and every time a new box of test strips was opened.

Resuscitation and supportive management were given according to the WHO guidelines [3]; i.e. quinine infusion at $10 \mathrm{mg} / \mathrm{kg}$ three times a day over 2-3 hours changed to oral quinine tablet when the patient could tolerate them, correction of hypoglycaemia with $10 \%$ glucose, termination of convulsions with intravenous diazepam if they were persisting for more than three minutes. Paracetamol was given every 6 hours until defervescence. Those with severe anaemia (haemoglobin $<7 \mathrm{~g} / \mathrm{dl}$ ) and respiratory distress were transfused with blood screened for hepatitis and HIV. Vital signs were measured every 15 minutes for the first hour, then every 2 hours until 24 hours, and thereafter every 6 hours until the discharge from the hospital. Baseline investigations were performed for every patient on admission and repeated when clinically indicated. These included levels of haemoglobin, serum urea, serum creatinine, and serum bilirubin as well as the white blood cell count.

\section{Statistics}

Data were entered into a computer database and SPSS software (SPSS Inc., Chicago, IL, USA) and double checked before analysis. Means (SD) and proportions for the socio-demographic and biochemical variables were calculated. ANOVA was used to compare the means (SD) between the different groups of severe malaria. Correlations between the different continuous clinical and biochemical variables were calculated. $P<$ 0.05 was considered significant. 


\section{Ethics}

The study received ethical clearance from the Research Board at the Faculty of Medicine, University of Khartoum.

\section{Results}

Among 222 pregnant women diagnosed with malaria at the two hospitals, 40 (18.0\%) fulfilled one or more of the WHO criteria for severe $P$. falciparum malaria. These were hypoglycaemia $(14 ; 35.5 \%)$, severe anaemia (12; 30\%), hypotension $(10 ; 25 \%)$, jaundice $(9 ; 22.5 \%)$, cerebral malaria $(6 ; 15 \%)$, repeated convulsions $(4 ; 10 \%)$, hyperparasitaemia $(4 ; 10.0 \%)$ and more than one manifestation $(9 ; 22.5 \%)$, Table 1 . Ten patients with severe anaemia received blood transfusion. Out the 12 patients with severe anaemia; three patients (25\%) had jaundice, one patient had hypotension and hyperparasitaemia (parasite count was 133333 rings/ $\mu$ ) and one patient had hypogylcaemia. The mean (SD) of the age, gravidity and gestational age of these 40 women were 28.4 (6.1) years, 3.5 (2.3) and 29.3 (6.7) weeks, respectively, Table 2. The parity ranged from 1 to 9 , (median $=2$ ) and $10(25 \%)$ of these women were primigravidae. None of the patients had used antimalarial chemoprophylaxis. There were no maternal deaths. All women were febrile. Different symptoms such as sweating, aches, vomiting and diarrhoea were observed among these women, Figure 2. None of the patients developed hypoglycaemia during quinine treatment. All patients started oral quinine tablet within two days.

While the mean (SD) presenting temperature was significantly lower in women presenting with hypoglycaemia [38.2(0.6) versus $38.8(0.7){ }^{\circ} \mathrm{C}, P=0.04$ ], other clinical and biochemical characteristics were not significantly different among women with different manifestations of severe $P$. falciparum malaria, Table 3 . The haemoglobin level was significantly lower in the anaemic group, Table 3. There was no significant correlation between blood glucose levels and temperature, haemoglobin levels and parasite counts, Table 4.

Table 1 presentations of severe $\boldsymbol{P}$. falciparum malaria at Kassala and Medani Maternity Hospitals, Sudan

\begin{tabular}{lll}
\hline $\begin{array}{l}\text { Criteria of severe } \boldsymbol{P} \text {. falciparum } \\
\text { malaria }\end{array}$ & $\begin{array}{l}\text { Number of } \\
\text { women }\end{array}$ & Percentage \\
\hline Hypoglycaemia & 14 & 35.5 \\
Severe anaemia & 12 & 30.0 \\
Hypotension & 10 & 25.0 \\
Jaundice & 9 & 22.5 \\
Cerebral malaria & 6 & 15.0 \\
Convulsions & 4 & 10.0 \\
Hyperparasitaemia & 4 & 10.0 \\
More than one complication & 9 & 22.5 \\
\hline
\end{tabular}

Table 2 presenting clinical and biochemical data of the pregnant women with severe $P$. falciparum malaria at Kassala and Medani Maternity Hospitals, Sudan

\begin{tabular}{ll}
\hline Variables & range, mean \pm SD \\
\hline Age, years & $28.4 \pm 6.1[18-40]$ \\
Gravidity & $3.5 \pm 2.3[1-9]$ \\
Gestational age, weeks & $29.3 \pm 6.7[14-38]$ \\
Duration of illness, days & $2.5 \pm 1.1[1-6]$ \\
Weight, kg & $59.3 \pm 7.1[43-75]$ \\
Temperature, ${ }^{\circ} \mathrm{C}$ & $38.5 \pm 0.7[37.8-40.0]$ \\
Haemoglobin, $\mathrm{gm} / \mathrm{dl}$ & $8.3 \pm 1.7[5-11.0]$ \\
White blood cells, cell/mm ${ }^{3}$ & $6300 \pm 2927[2700-14000]$ \\
Parasite count, rings/ $\mu \mathrm{l}$ & $30717 \pm 5270[2080-335242]$ \\
Blood glucose, $\mathrm{mg} / \mathrm{dl}$ & $71.4 \pm 3.0[29-120]$ \\
serum bilirubin, $\mathrm{mg} / \mathrm{dl}$ & $2.0 \pm 0.7[1-3]$ \\
Serum creatinine, $\mathrm{mg} / \mathrm{dl}$ & $1.0 \pm 0.2[0.7-2.0]$ \\
\hline
\end{tabular}

\section{Discussion}

The main findings of the present study were that hypoglycaemia and severe anaemia were the predominant presenting manifestations of severe $P$. falciparum malaria observed during pregnancy in this epidemiological setting. With the exception of lower temperatures, other clinical and biochemical criteria were not different among women with different criteria of severe P. falciparum malaria. Previously, severe anaemia and jaundice had been observed as the presenting manifestations of severe $P$. falciparum malaria in pregnant women in central and eastern Sudan $[5,14]$. In neighbouring Ethiopia, cerebral malaria, convulsions, altered mental state and prostration were the common manifestations of severe malaria observed in pregnant women [15]. Generally, pregnant women are more susceptible to severe malaria and hypoglycaemia than their non-pregnant peers $[3,7]$. The glucose metabolism during malaria infection is affected by several factors, including drug treatment, fever, parasite metabolism, hormonal changes, cytokines, fasting and gastrointestinal disturbances $[16,17]$. It has been reported that patients with severe malaria-induced hypoglycaemia have higher mortality rates [18]. Thus, the recognition of patients with falciparum malaria and hypoglycaemia by blood glucose estimation at the time of admission could significantly affect the ultimate outcome. Interestingly, some comatose patients regained consciousness with intravenous fluid infusion of $25 \%$ dextrose only without receiving any specific antimalarial treatment [18]. None of these patients developed hypoglycaemia after quinine treatment. In this study blood glucose was investigated at base line, two hours following quinine and if hypoglycaemia was clinically suspected. Ideally, blood glucose should be investigated every four hours if possible especially in comatose patients according to the WHO guidelines [19]. 


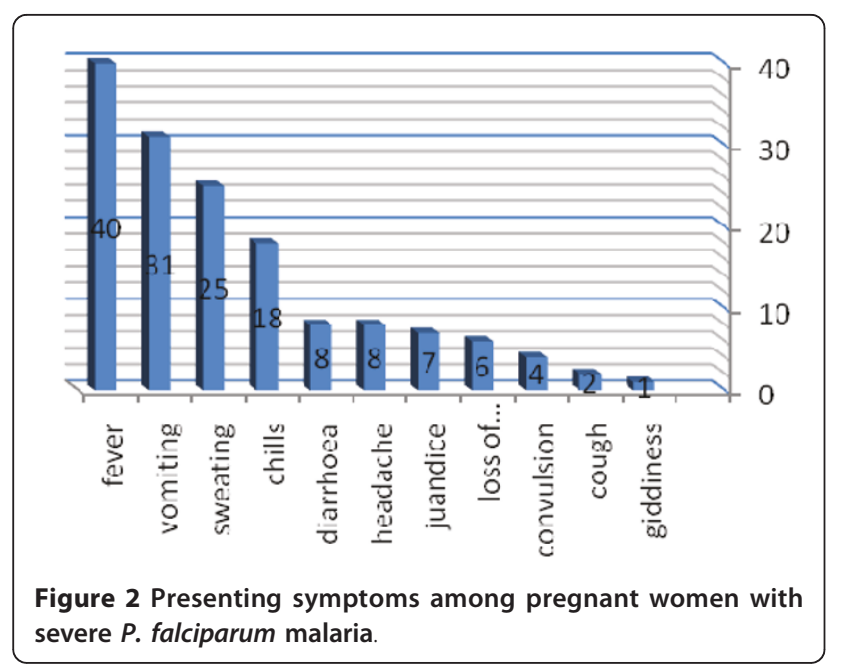

Therefore, this is one of the limitations of this study where quinine -induced hypoglycaemia was not investigated as should be. Previously, only one out of 33 pregnant Sudanese women developed hypoglycaemia following quinine treatment for severe $P$. falciparum malaria [5]. Hyperinsulinaemic hypoglycaemia is the most important adverse effect in the quinine treatment of severe malaria which is particularly common in pregnancy $(50 \%$ of quinine-treated women with severe malaria in late pregnancy) $[20,21]$. Intravenous artesunate is superior to quinine in the treatment of severe malaria [22]. Compared to intravenous quinine, intravenous artesunate has been shown to have; a lower risk of hypoglycaemia, significantly reduce the risk of death from severe malaria, and it is not requiring rate controlled infusion or cardiac monitoring [19]. Patients in this series were in their second and third trimester of pregnancy; therefore intravenous artesunate would have been given to these women instead of quinine. However, intravenous artesunate is not yet registered and available in Sudan.

In the present study, $12(30 \%)$ and $6(15 \%)$ patients presented with severe anaemia and cerebral malaria, respectively. Cerebral malaria and severe malarial anaemia are two major syndromes causing malariarelated mortality [4]. The pattern of these two severe forms varies depending on the intensity of transmission; cerebral malaria is more common in older children in areas with lower intensity of transmission, whereas severe malarial anaemia is often seen in children below two years of age in areas with intense transmission [4]. Maternal anaemia and malaria have been reported in areas of unstable malaria transmission in Thailand and in Ethiopia, as well as in areas of stable malaria transmission [23,24]. Regardless of the transmission level and the level of pre-pregnancy immunity against malaria, maternal anaemia remains the most frequent consequence of malaria during pregnancy [25]. Interestingly, we have recently observed a high prevalence of anaemia in pregnant women in these two hospitals and anaemic women were at a higher risk of stillbirth and low birth weight deliveries [26-29]. Interestingly, seven out 12 patients in the current study had severe anaemia without evidence of multiorgan dysfunction or other manifestations of severe of malaria. Although, these patients fulfilled the WHO criteria for severe malaria [3], perhaps some of these women had severe anaemia and concurrent uncomplicated malaria rather than severe $P$. falciparum malaria. Thus, in such situation these patients would have received blood transfusion and artemisinins combination therapy rather than quinine treatment.

There were no maternal deaths in this study, early diagnosis; prompt effective treatment could explain this observation. These women would appear to represent a milder spectrum of disease e.g. severe anaemia and hypotension. It have been shown that, within the broad definition of severe P. falciparum malaria there are syndromes associated with mortality rates that are lower (e.g. severe anaemia) and higher (cerebral malaria and metabolic acidosis) [19]. We previously observed that malaria was one of the main causes of high maternal mortality in these two hospitals [9,30]. Maternal mortality is approximately $50 \%$ in pregnant women with severe $P$. falciparum malaria, which is higher than in non-

Table 3 comparison of clinical and biochemical data in the subgroups of the pregnant women with severe $P$. falciparum malaria at Kassala and Medani Maternity Hospitals, Sudan

\begin{tabular}{|c|c|c|c|c|}
\hline Variable & Hypoglycemic group $(N=14)$ & Anaemic group $(N=12)$ & $\begin{array}{l}\text { Other group } \\
(N=14)\end{array}$ & $P$ \\
\hline Age, years & $27.1(8.1)$ & $30.1(5.9)$ & $28.8(4.4)$ & 0.1 \\
\hline Gravidity & $3.8(2.9)$ & $3.6(2.7)$ & $3.5(1.9)$ & 0.3 \\
\hline Gestational age, weeks & $26.9(6.4)$ & $29.2(8.4)$ & $32.0(4.5)$ & 0.6 \\
\hline Weight, kg & $59.0(6.6)$ & $61.3(7.7)$ & $58.3(7.6)$ & 0.2 \\
\hline Temperature, ${ }^{\circ} \mathrm{C}$ & $38.2(0.6)$ & $38.5(0.5)$ & $38.8(0.7)$ & 0.04 \\
\hline Haemoglobin, gm/dl & $8.4(1.4)$ & $6.2(0.7)$ & $9.2(1.1)$ & 0.001 \\
\hline parasite count, ring $/ \mu$ & $32057(62169.1)$ & 27191(37961) & 29244(3633) & 0.9 \\
\hline
\end{tabular}


Table 4 Correlation between various clinical and biochemical measures including haemoglobin

\begin{tabular}{lllllll}
\hline Variable & \multicolumn{2}{c}{ Temperature } & \multicolumn{2}{c}{ Blood glucose } & \multicolumn{2}{c}{ Parasite count } \\
& $\mathbf{r}$ & $\boldsymbol{P}$ & $\mathbf{r}$ & $\boldsymbol{P}$ & $\mathbf{r}$ & $\boldsymbol{P}$ \\
\hline Haemoglobin & 0.242 & 0.1 & 0.146 & 0.3 & 0.129 & 0.4 \\
Temperature & & & 0.290 & 0.07 & 0.013 & 0.9 \\
Blood glucose & & & & & 0.079 & 0.6 \\
\hline
\end{tabular}

pregnant adults [19]. The other limitation of this work is that we could not follow up these women and investigate/report the maternal and perinatal outcomes, and compare them to women with uncomplicated P. falciparum malaria and healthy controls.

\section{Conclusion}

Preventive measures for pregnant women such as insecticide-treated bednets and chemoprophylaxis may be beneficial in areas of unstable malaria transmission. Early detection and prompt treatment of severe malaria, especially in pregnant women with hypogylcaemia, are needed.

\section{Acknowledgements}

Authors are very grateful to the women who participated in the study and to all the staff of Medani and Kassala maternity hospitals. This work was funded by The National Fund for promotion of Medical Service, Khartoum, Sudan.

\section{Author details}

${ }^{1}$ Faculty of Medicine, Kassala University, Sudan. ${ }^{2}$ University of Geizera, Wad Medani, Sudan. ${ }^{3}$ Faculty of Medicine, University of Khartoum, Sudan.

\section{Authors' contributions}

AAA and EME carried out the study and participated in the statistical analysis and procedures. MMM carried out the biochemical tests. IA and MIE coordinated and participated in the design of the study, statistical analysis and the drafting of the manuscript. All the authors read and approved the final version.

\section{Competing interests}

The authors declare that they have no competing interests.

Received: 15 March 2011 Accepted: 23 May 2011

Published: 23 May 2011

\section{References}

1. World Health Organization: Strategic Framework for Malaria Control during Pregnancy in the WHO Africa Region, Brazzaville. WHO Regional Office for Africa 2004, AFR/MAL/04/01.

2. Tagbor H, Bruce J, Browne E, Greenwood B, Chandramohan D: Malaria in pregnancy in an area of stable and intense transmission: is it asymptomatic? Trop Med Inter Health 2008, 13:1018-1021.

3. WHO: Severe and complicated malaria. Trans R Soc Trop Med Hyg 2000, 94(Suppl 1):1-90.

4. Greenwood BM: The epidemiology of malaria. Ann Trop Med Parasitol 1997, 91:763-769.

5. Adam I, Mirghani OA, Saed OK, Ahmed SM, Mohamadani AA, Ahmed HM, Mackenzie CD, Homeida MM, Elbashir Ml: Quinine therapy in severe Plasmodium falciparum malaria during pregnancy in Sudan. East Mediterr Health J 200, 10:159-66.
6. Himeidan YE, Elbashir Ml, Adam I: Attractiveness of pregnant women to the malaria vector, Anopheles arabiensis, in Sudan. Ann Trop Med Parasitol 2004, 98:631-3.

7. Luxemburger C, Ricci F, Nosten F, Raimond D, Bathet S, White NJ: The epidemiology of severe malaria in an area of low transmission in Thailand. Trans R Soc Trop Med Hyg 1997, 91:256-262.

8. ElGhazali G, Adam I, Hamad AA, Elbashir MI: Malaria and pregnancy in an area of unstable transmission in eastern Sudan. East Mediterr Health $J$ 2003, 9:571-580.

9. Elhassan EM, Mirghani OA, Adam I: High maternal mortality and stillbirth in the Wad Medani Hospital, Central Sudan, 2003-2007. Trop Doct 2009, 39:238.

10. Adam I, Elbashir Ml: Maternal death due to severe pulmonary oedema caused by falciparum malaria: a case report. East Mediterr Health J 2004, 10:685-7.

11. Bader E, Alhaj AM, Hussan AA, Adam I: Malaria and stillbirth in Omdurman Maternity Hospital, Sudan. Int J Gynaecol Obstet 2010, 109:144-6.

12. Malik EM, Atta H Y, Weis M, Lang A, Puta C, Lettenmaier C: Sudan Roll Back Malaria Consultative Mission: Essential Actions to Support the Attainment of the Abuja Targets. Sudan RBM Country Consultative Mission Final Report. Geneva Roll Back Malaria Partnership 2004.

13. Adam I, Khamis A H, Elbashir Ml: Prevalence and risk factors for malaria in pregnant women of eastern Sudan. Malar J 2005, 4:8.

14. Adam I, Ali DM, Elbashir MI: Manifestations of falciparum malaria in pregnant women of Eastern Sudan. Saudi Med J 2004, 25:1947-50.

15. Mengistu G, Diro E, Kassu A: Outcomes of pregnancy in severe malaria with emphasis on neurological manifestations in Gondar Hospital northwest Ethiopia. Ethiop Med J 2006, 44:321-30.

16. Davis TM, Looareesuwan S, Pukrittayakamee S, Levy JC, Nagachinta B, White NJ: Glucose turnover in severe falciparum malaria. Metabolism 1993, 42:334-340.

17. Davis TM, Binh TQ, Thu le TA, Long TT, Johnston W, Robertson K, Barrett PH: Glucose and lactate turnover in adults with falciparum malaria: effect of complications and antimalarial therapy. Trans $R$ Soc Trop Med Hyg 2002, 96:411-417.

18. Kochar DK, Thanvi I, Kumawat BL, Agarwal N: Importance of blood glucose level at the time of admission in severe and complicated malaria. $J$ Assoc Physicians India 1998, 46:923-5.

19. WHO. Guidelines for the treatment of malaria , 22010 [http://www.who.int/ malaria/publications/atoz/9789241547925], Languages: English ISBN: 9789241547925.

20. Looareesuwan S, Phillips RE, White NJ, Kietinun S, Karbwang J, Rackow C, Turner RC, Warrell DA: Quinine and severe falciparum malaria in late pregnancy. Lancet 1985, 2(8445):4-8.

21. White NJ, Looareesuwan S, Warrell DA: Quinine and quinidine: a comparison of EKG effects during the treatment of malaria. J Cardiovasc Pharmacol 1983, 5:173-175.

22. Sinclair D, Donegan S, Lalloo DG: Artesunate versus quinine for treating severe malaria. Cochrane Database Syst Rev 2011, 3:CD005967.

23. Nosten F, Ter Kuile FO, Maelankirri L, Decludt B, White NJ: Malaria during pregnancy in an area of unstable endemicity. Trans $R$ Soc Trop Med Hyg 1991, 85:424-429.

24. Newman RD, Hailemariam A, Jimma D, Degefie A, Kebede D, Rietveld AC, et al: Burden of malaria during pregnancy in areas of stable and unstable transmission in Ethiopia during a non-epidemic year. J Infect Dis 2003, 187:1765-1772

25. Menendez C: Malaria during pregnancy: A priority area of malaria research and control. Parasitol Today 1995, 187:178-183.

26. Mohamed AA, Ali AA, Ali NI, Abusalama EH, Elbashir MI, Adam I: Zinc, parity, infection, and severe anemia among pregnant women in Kassala, Eastern Sudan. Biol Trace Elem Res 137(3):225-230.

27. Bushra M, Elhassan EM, Ali NI, Osman E, Bakheit KH, Adam II: Anaemia, zinc and copper deficiencies among pregnant women in central Sudan. Biol Trace Elem Res 2010, 137:255-61.

28. Ali AA, Adam I: Anaemia and Stillbirth in Kassala Hospital, Eastern Sudan. J Trop Pediatr 2011, 57(1):62-4.

29. Elhassan EM, Abbaker AO, Haggaz AD, Abubaker MS, Adam I: Anaemia and low birth weight in Medani, Hospital Sudan. BMC Res Notes 2010, 28(3):181. 
30. Ali AA, Adam I: Lack of antenatal care, education, and high maternal mortality in Kassala hospital, eastern Sudan during 2005-2009. J Matern Fetal Neonatal Med 2011.

doi:10.1186/1756-3305-4-88

Cite this article as: Ali et al:. Hypoglycaemia and severe plasmodium falciparum malaria among pregnant sudanese women in an area characterized by unstable malaria transmission. Parasites \& Vectors 2011 4:88.

Submit your next manuscript to BioMed Central and take full advantage of:

- Convenient online submission

- Thorough peer review

- No space constraints or color figure charges

- Immediate publication on acceptance

- Inclusion in PubMed, CAS, Scopus and Google Scholar

- Research which is freely available for redistribution

Submit your manuscript at www.biomedcentral.com/submit 